\title{
Hypothermic circulatory arrest in cerebral aneurysm surgery
}

\author{
Prandini $\mathrm{MN}^{1 *}$, Nonato $\mathrm{MB}^{2}$ and Balestrieri $\mathrm{JVL}^{3}$ \\ ${ }^{1}$ Professor of Neurosurgery Federal University of São Paulo, Brazil \\ ${ }^{2}$ Medical student, UNIFAI, Adamantina, Brazil \\ ${ }^{3}$ Medical Student, UNILAGO, São José do Rio Preto, Brazil
}

\begin{abstract}
Moderate hypothermia $\left(30^{\circ} \mathrm{C}-33^{\circ} \mathrm{C}\right)$ has been widely used in cerebral aneurysms surgeries with excellent results. However not all aneurysms could be safely operated on since a small number, because of its special conditions, requires a longer period without arterial circulation

The advances of cardiac surgery with the use of profound systemic hypothermia to preserve organ function during cessation of the circulation termed deep hypothermic circulatory arrest (DHCA) permitted the approach of giant and complexes aneurysms with the preservation of brain function.
\end{abstract}

\section{Introduction}

It is a fact that the indications for treatment of cerebral aneurysm by the endovascular technique have been increasing along the years. However, it is also a fact that the endovascular approach is not able to manage all brain aneurysms at present time. Therefore, neurosurgeons must be prepared to treat those cases at the present time and some of them in the future.

\section{Historical}

The therapeutic effect of low temperature is known for more than 2.500 years, when the Egyptian used cold to treat wounds and inflammations and the Greeks used snow to stop bleeding or reduce the edema in wounded soldiers.

Canadian surgeon Wilfred Bigelow demonstrated in animal models that the length of time the brain could survive could be extended from 3 minutes to 10 minutes by cooling to $30^{\circ} \mathrm{C}$ before circulation was stopped. Surface cooling with cold rubber blankets or an iced water bath permitted more prolonged procedures requiring cardiac arrest. However, the incidence of death and serious complications greatly reduced their applications [1]. Subsequent reports of the use of more profound degrees of hypothermia highlighted the risks of cold-induced ventricular fibrillation and coagulopathic haemorrhage $[2,3]$.

In 1959, Ross and Sir Russel Brock, from Guy's Hospital London, declared "deep hypothermia by means of a heart lung bypass machine or a differential cooling technique holds promise of longer safe periods of intra cardiac surgery in the future" [4]. Following this pronouncement, there was a wave of experiments in the use of cardiopulmonary bypass with hypothermia. The modern era of cardiac surgery, heralded by the introduction of cardiopulmonary bypass $(\mathrm{CPB})$ into clinical practice provided surgeons with the means to both cool and warm patients while maintaining organ perfusion. In some situations, however, the nature of the surgery proposed necessitates complete cessation of the circulation. The use of profound systemic hypothermia to preserve organ function during cessation of the circulation is termed deep hypothermic circulatory arrest (DHCA) [5].

The technique provides excellent operating conditions while reducing the consequences of organ ischemia. As the brain is the organ most susceptible to ischemia during circulatory arrest, it follows that other organs will also be protected by this strategy. Griepp, in 1975, demonstrated that the technique offered a practical and safe approach for aortic arch surgery. Since then DHCA has remained the mainstay of cerebral protection during complex cardiac, vascular, neurological, and urological procedures [6].

\section{In neurosurgery}

In the field of neurosurgery moderate hypothermia $\left(30^{\circ} \mathrm{C}-33^{\circ} \mathrm{C}\right)$ has been widely used with excellent results in most of cases of aneurysm surgery.

Considering that ischemic lesion is proportional to time of ischemia and consume of oxygen by the organs, several attempts to reduce oxygen consumption led to the conclusion that hypothermia was the most efficient protective element of brain parenchyma. Zager et al. in animals retina [7], which metabolism is similar to the brain parenchyma, could demonstrate that lowering the temperature by 3 degrees the consumption of oxygen was reduced by $20 \%$ and by 6 degrees the reduction reached $37 \%$.

In 2002, in order to prove the possibility of neuroprotection that could be provided by moderate hypothermia Prandini et al. [8] after the removal of an area of $9 \mathrm{~cm}$ square of the cranial bone of rabbits

Correspondence to: Prandini MN, Rua dos Crisântemos, São Paulo SP BRAZIL CEP; E-mail: mnprandini@uol.com.br; Tel: +5511 55818761/ 5511991156830

Key words: hypothermia, hypothermic circulatory arrest, aneurysm surgery

Received: December 04, 2017; Accepted: December 22, 2017; Published: December 29, 2017 
proceeded to coagulation of the left middle cerebral artery causing an important ischemia in the area nourished by this vessel. The scalp was then sutured, and one ice pack was applied for 120 minutes covering the cranial vault in order to keep the brain temperature around 30 degrees. After 3 days the animals were sacrificed, and the brains were removed and stained with TTC a special dye for the evaluation of mitochondria. The ischemic area was measured. The infarcted area was: $805,40 \mathrm{~mm}^{3}$ in animals without protection and $204.70 \mathrm{~mm}^{3}$ in animals that were protected. The results could demonstrate that the hypothermia was effective in promoting neuroprotection (Figure 1).

It is well known that the neuroproctive mechanisms obtained by using hypothermia are multifactorial. Cerebral ischemia is accompanied by a marked inflammatory reaction that is initiated by ischemia-induced expression of cytokines, adhesion molecules, and other inflammatory mediators, including prostanoids and nitric oxide. Considering these facts, remained the need to prove that hypothermia could also present a neuroprotective factor by reducing the inflammatory process developed in the ischemic conditions [9].

Prandini et al. carried out a study with brains of rats in which an inflammatory reaction was provoked by means of the application on the cerebral parenchyma of a powerful inflammatory substance. It was demonstrated that local hypothermia $\left(30^{\circ} \mathrm{C}\right)$ applied for 120 minutes, with ice packs, considerably reduced the number of inflammatory cells in animals in which the process was implemented. The animal's corporal temperature of $37^{\circ} \mathrm{C}$ was maintained with the use of thermic blankets (Figure 2 and 3).

Thus, over the last 20 years, local hypothermia has become routine in our department for cerebral aneurysm surgeries.

In some rare cases, due to its large volume and complexity, the aneurysm approach can cause severe damage to brain. In these cases, it is necessary to remove part of the wall of the aneurysm prior to its neck clipping. Circulatory arrest is essential.
After the development of deep hypothermia and circulatory arrest for cardiothoracic procedures in the late 1950s, this technique was adopted by several neurosurgeons as an aid to complex cranial surgery. Woodhall and colleagues described its first use for a neurosurgical procedure in 1960 [10].

Although their case did not involve a cerebrovascular procedure, the technique was subsequently used for the surgical treatment of cerebrovascular lesions, especially complex and giant aneurysms. Subsequent improvements in the technique and advances in the equipment designed for a cardiopulmonary bypass have led to its more widespread use starting in the 1980s. Since that time hypothermic circulatory arrest has been described in several reports as a safe and useful tool in the treatment of large and giant aneurysms [11].

According to Connoly et al. [12], the majority of patients will tolerate $30 \mathrm{~min}$ of circulatory arrest at $18^{\circ} \mathrm{C}$ without significant neurological impairment. Although not routinely we can consider the use of DHCA in cases of patients aged less than 75 years old, and who do not have severe cardiac or systemic diseases. The circulatory arrest may not overpass 30 minutes which we consider a very satisfactory time for the aneurysm management. Our surgical steps: wide craniotomy, brain dissection until the aneurysm is reached. The DHCA starts and after the circulatory arrest, most of the aneurysm wall is removed. Then, the aneurysm neck can be better dissected and clipped. The circulation restarts and a careful evaluation of the brain circulatory conditions are performed, before closing the craniotomy.

\section{Discussion}

Giant and complex aneurysms are still a challenge for surgeons. Moderate hypothermia cannot protect some rare cases where aneurysms approaches require a removal of part of its wall in order to permit a wide reduction of the lesion before it can be conveniently treated. The association with cardiac surgery has permitted neurosurgeons to carry out safely procedures that were "forbidden" until a few years ago.

\section{NO PROTECTION}

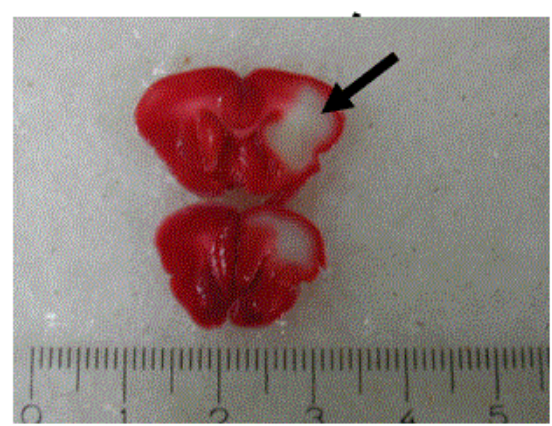

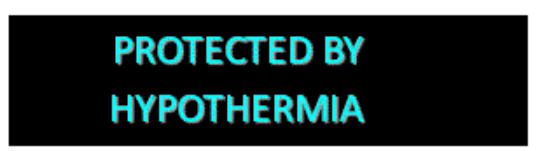

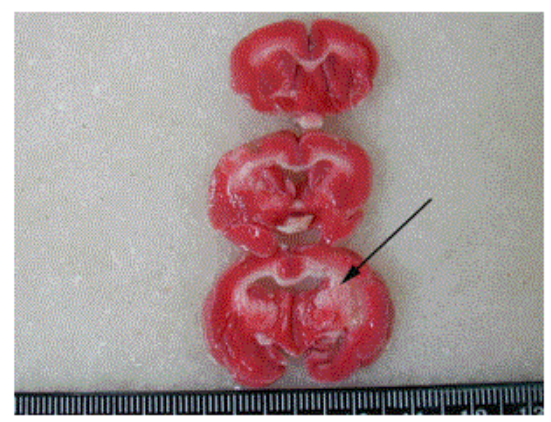

\section{INFARCTED AREA IN $\mathrm{mm} 3$}
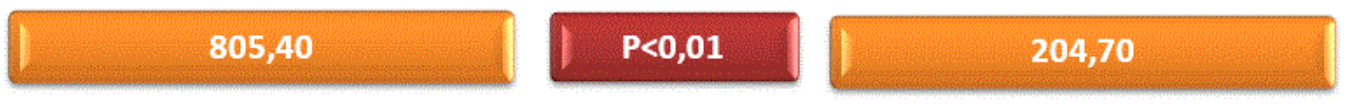

Figure 1. Sections of brains of rabbits. Arrow- infarcted area. 

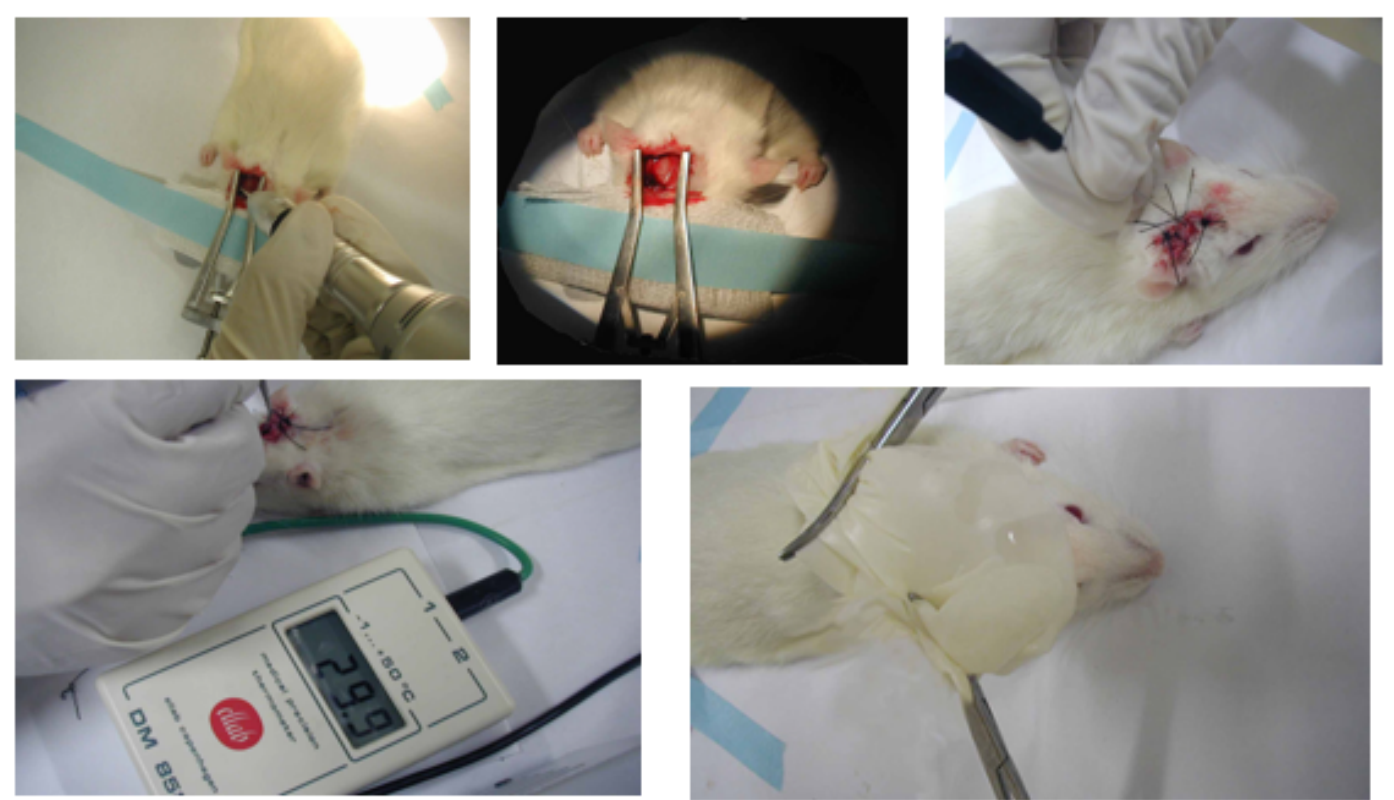

Figure 2. Inflammatory lesion in rats brain
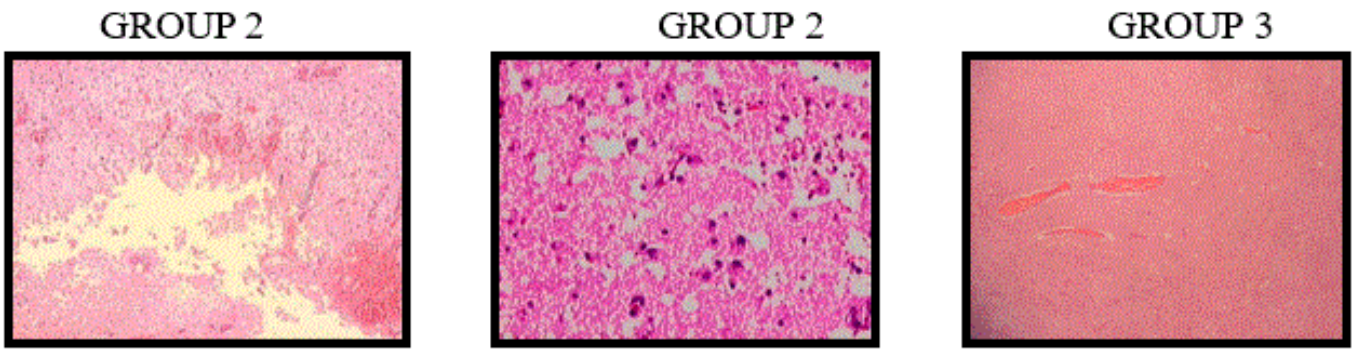

\begin{tabular}{|c|c|c|}
\hline$\frac{\text { PMNL / }}{\text { FIELD }}$ & $\begin{array}{l}\text { GROUP } \\
2\end{array}$ & $\begin{array}{l}\text { GROUP } \\
3\end{array}$ \\
\hline 1 & 98 & 30 \\
\hline 2 & 88 & 38 \\
\hline 3 & 96 & 28 \\
\hline 4 & 72 & 24 \\
\hline 5 & 80 & 38 \\
\hline 6 & 72 & 32 \\
\hline 7 & 88 & 34 \\
\hline 8 & 72 & 40 \\
\hline 9 & 88 & 24 \\
\hline 10 & 96 & 32 \\
\hline
\end{tabular}

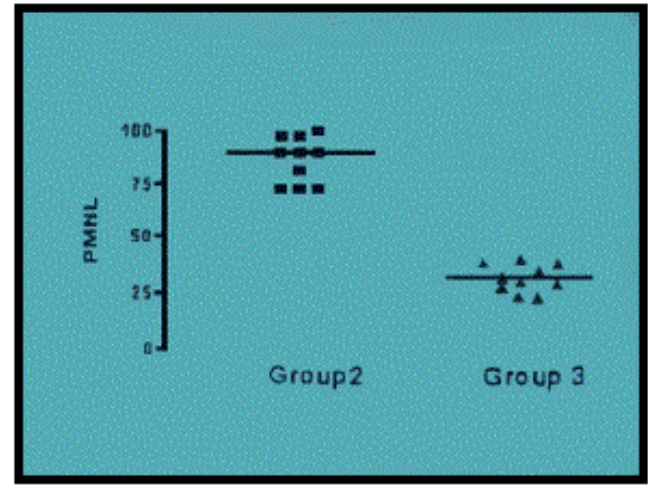

$\mathrm{p}<0,05$

Figure 3. Brain of Rats H.E.

\section{Conclusion}

Association of cardiac surgery with neurosurgery was very effective in allowing the approach of complex aneurysms fact that up to a few years ago was considered as unattainable.

\section{References}

1. Sealy WC, Brown IW Jr, Young WG Jr (1958) A report on the use of both extracorporeal circulation and hypothermia for open heart surgery. Ann Surg 147: 603-613. [Crossref]
2. Cooley DA (2013) A Brief History of Aortic Aneurysm Surgery. Aorta (Stamford) 1: 1-3. [Crossref]

3. Ziganshin BA, Elefteriades JA (2013) Deep hypothermic circulatory arrest. Ann Cardiothorac Surg 2: 303-315. [Crossref]

4. BROCK R, ROSS DN (1955) Hypothermia. III. The clinical application of hypothermic techniques. Guys Hosp Rep 104: 99-113. [Crossref]

5. Rimmer L, Fok M, Bashir M (2014) The History of Deep Hypothermic Circulatory Arrest in Thoracic Aortic Surgery. AORTA (Stamford) 2: 129-134. [Crossref] 
6. Naef A (2004) The mid-century revolution in thoracic and cardiovascular surgery: part 4. Interact Cardiovasc Thorac Surg 3: 213-221. [Crossref]

7. Zager EL, Ames A III (1998) Reduction of cellular energy requirements. Screening for agents that may protect against CNS ischemia. J Neurosurg 69: 568-579. [Crossref]

8. Prandini MN, Lacann SN, Valente PR, Stavale JN (2002) Regional mild hipotermia inthe protection of the ischemic brain. Acta Cirúrgica Brasileira.17: 232-235.

9. Prandini MN, Filho AN, Lapa AJ, Stavale JN (2005) Mild hypothermia reduces polymorphonuclear leukocytes infiltration in induced brain inflammation. Arq Neuropsiquiatr 63:779-784. [Crossref]
10. Woodhall B, Sealy WC, Hall KD, Floyd WL (1960) Craniotomy under conditions of quinidine-protected cardioplegia and profound hypothermia. Ann Surg 152: 37-44. [Crossref]

11. Rothoerl RD, Brawanski A (2006) The history and present status of deep hypothermia and circulatory arrest in cerebrovascular surgery. Neurosurg Focus 20: E5. [Crossref]

12. Conolly S, Arrowsmith JE, Klein AA (2010) Deep hypothermic circulatory arrest, Continuing Education in Anaesthesia Critical Care \& Pain. 10: 138-142.

Copyright: $@ 2018$ Prandini MN. This is an open-access article distributed under the terms of the Creative Commons Attribution License, which permits unrestricted use, distribution, and reproduction in any medium, provided the original author and source are credited. 